\title{
Synthesis of Metastable Au-Fe Alloy Using Ordered Nanoporous Silica as a Hard Template
}

\author{
Paskalis Sahaya Murphin Kumar ${ }^{1,2}$, Thiripuranthagan Sivakumar ${ }^{1}$, Takeshi Fujita ${ }^{3}$, \\ Ramasamy Jayavel ${ }^{4}$ and Hideki Abe ${ }^{2, *}$ \\ 1 Department of Applied Science and Technology, Anna University, Chennai 600025, India; \\ marpinindian@gmail.com (P.S.M.K.); tssivakumar@yahoo.com (T.S.) \\ 2 Center for Green Research on Energy and Environmental Materials, National Institute for Materials Science, \\ Namiki 1-1, Tsukuba, Ibaraki 305-0044, Japan \\ 3 WPI Advanced Institute for Materials Research, Tohoku University, Sendai 980-8577, Japan; \\ tfujita@wpi-aimr.tohoku.ac.jp \\ 4 Centre for Research, Anna University, Chennai 600025, India; rjvelsms@gmail.com \\ * Correspondence: Abe.Hideki@nims.go.jp; Tel.: +81-298-604-803
}

Received: 24 November 2017; Accepted: 23 December 2017; Published: 30 December 2017

\begin{abstract}
Nanoporous Au-Fe alloy was synthesized via a wet chemistry route using ordered nanoporous silica as a hard template. The nanoporous Au-Fe consisted of aligned arrays of nanopores that were uniform in composition and ordered in hexagonal lattice, whereas Au-Fe nanoparticles synthesized without templates exhibited broad dispersions in the chemical composition and/or particle size. Nanoporous Au-Fe has potential for applications as catalysts and/or adsorbents because of the large specific surface area of $81.2 \mathrm{~m}^{2} \cdot \mathrm{g}^{-1}$ and high pore volume of $0.56 \mathrm{~cm}^{3} \cdot \mathrm{g}^{-1}$.
\end{abstract}

Keywords: gold; iron; metastable; alloy; nanoporous; ordered nanoporous silica

\section{Introduction}

Alloy materials are widely used for day-to-day life. Copper-zinc alloys for corrosion-tolerant piping, gold-silver alloys for hardened jewelry wires, and titanium-aluminum alloys for biocompatible implants. Important infrastructures including bridges and railways are supported by hardened steels consisting of iron, chromium, and different metal additives. In the past few decades, alloy materials having nanometer pores and/or particle sizes, namely alloy nanomaterials, have been attracting attention because of their prominent performance as plasmonic sensors and catalysts for a broad range of energy/environmental applications [1].

Alloy nanomaterials are produced via either dry processes such as sputtering of alloy targets or wet chemistry routes including co-reduction of soluble metal precursors such as organometallic compounds and/or metal halides [2]. The wet chemistry routes are superior to the dry processes in terms of the ability to precisely control the pore-size- and/or particle-size distribution [3]. Monodispersed Au-Ag nanoparticles were prepared by co-reduction of Gold [1] chloride trihydrate and silver acetate in aqueous solution containing oleylamine as a surfactant, of which particle-size distribution was within $6 \mathrm{~nm}$ to $13 \mathrm{~nm}$ [4]. Nanoporous Pd-Pt alloy materials were synthesized in aqueous solution by soft-template methods, where self-assembled micelles served as a soft template to realize a monodispersed pore diameter [5].

However, current wet chemistry routes face a challenge of low chemical homogeneity of the targeted alloy nanomaterials. The wet chemistry synthesized alloy nanomaterials are prone to phase segregation especially when the constituent metals are much different in reduction potentials: one of the metal precursors with a low reduction potential readily forms a core, on which the other metal precursor with a high reduction potential precipitates to develop core-shell structures [6]. Post-annealing 
of such as-prepared alloy nanomaterials can improve the compositional uniformity because of promoted atomic interdiffusion but often lead to uncontrollable broadening in the particle/pore-size distribution due to particle agglomeration $[7,8]$. Moreover, nanomaterials of thermodynamically unstable (metastable) alloys are hardly materialized by post-annealing because phase separations can be promoted more than atomic interdiffusion at elevated temperatures.

Herein, we report that ordered nanoporous silica materials (SBA15) can serve as an efficient hard template for the synthesis of metastable alloy nanomaterials consisting of metal elements with widely different reduction potentials (Scheme 1) [9]. The nanoporous template absorbs and disperses different metal precursors on the pore surface. The dispersed metal precursors are then reduced with chemical reagents to precipitate uniform alloy layers over the template. The nanoporous template is finally dissolved in solution to yield the desired alloy with a skeletal porous structure replicated from the template.

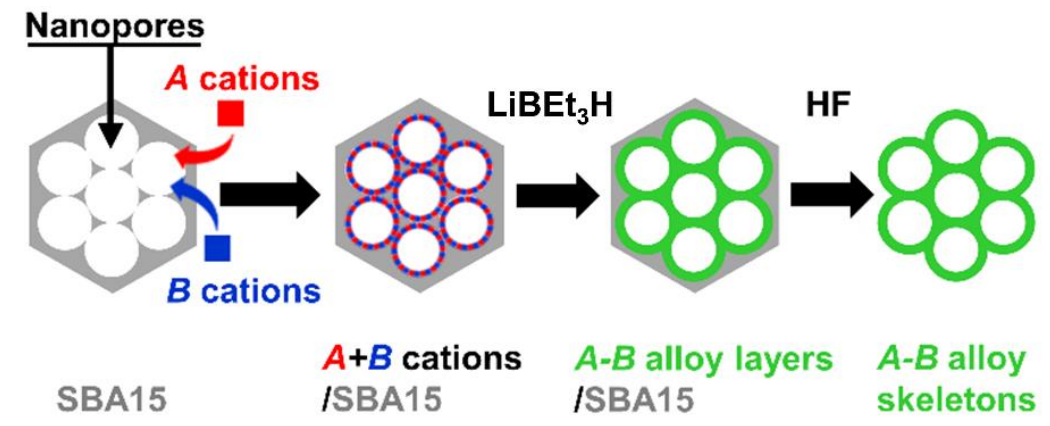

Scheme 1. Hard-template synthesis of skeletal nanoporous alloys via wet chemistry routes.

We demonstrate that this hard-template synthesis is successfully applicable to the gold $(\mathrm{Au})$-iron [10] alloy system although it is acknowledged that $\mathrm{Au}_{1-x} \mathrm{Fe}_{x}(0.5<x<0.9)$ is a thermodynamically metastable alloy that exhibits a phase separation at $750{ }^{\circ} \mathrm{C}$ or higher temperatures in ambient condition [11,12]. Gold chloride $\left(\mathrm{AuCl}_{3}\right.$, Sigma-Aldrich Japan, Tokyo, Japan) and iron acetylacetonate (Fe(II)acac) (Sigma-Aldrich Japan, Tokyo, Japan) were first dissolved in aprotic solvent containing SBA15 powder. Superhydride (Lithium Triethylborohydride in tetrahydrofuran) (Sigma-Aldrich Japan, Tokyo, Japan) was then added to the solution to reduce the $\mathrm{Au}(\mathrm{III})$-and $\mathrm{Fe}(\mathrm{II})$ precursors and precipitate nanometer-thick Au-Fe alloy layers over the pore surface of the template. The template was finally dissolved in hydrofluoric acid solution (Sigma-Aldrich Japan, Tokyo, Japan) to obtain the targeted Au-Fe nanomaterials having a nanoporous structure and large specific surface area. Powder X-ray diffractometry ( $p$ XRD) (Malvern PANalytical, Tokyo, Japan), hard X-ray photoemission spectroscopy (HAXPES) (Scienta Omicron, Tokyo, Japan), Brunauer-Emmett-Teller (BET) (Micromeritics Kashiwa, Japan) physisorption and scanning transmission electron microscopy (STEM) (JEOL, Tokyo, Japan) demonstrated that the obtained Au-Fe alloy nanomaterials had a uniform composition and an ordered nanoporous structure, which was inherited from the SBA15 hard template.

\section{Materials and Methods}

\subsection{Sample Preparation}

An aliquot of $200 \mathrm{mg}$ of SBA-15 was added to $35 \mathrm{~mL}$ of diethyleneglycol dimethyl (Sigma-Aldrich Japan, Tokyo, Japan) ether (diglyme) (Sigma-Aldrich Japan, Tokyo, Japan) and continuously stirred for $6 \mathrm{~h}$, then $50 \mathrm{mg}$ of $\mathrm{AuCl}_{3}$ and $106 \mathrm{mg}$ of $\mathrm{Fe}(\mathrm{II})$ acac were added to the solution and stirred for $1 \mathrm{~h}$ more. An aliquot of $2.2 \mathrm{~mL}$ of super hydride (1 M in tetrahydrofuran) (Sigma-Aldrich Japan, Tokyo, Japan) was finally added to the solution. The color of the solution turned from dark brown to turbid black upon addition of super hydride. The solution was allowed for stirring overnight and centrifuged to collect black powder as the final product. All of these procedures were carried out in 
dry Ar atmosphere in a glove box (mBraun, Garching, Germany). The collected black powder was exposed to air, washed with diglyme (Sigma-Aldrich Japan, Tokyo, Japan) and hexane (Sigma-Aldrich Japan, Tokyo, Japan), and finally heated at $700{ }^{\circ} \mathrm{C}$ in a vacuum-sealed quartz tube for $6.5 \mathrm{~h}$. The sample was immersed in 5\% aqueous HF solution (Sigma-Aldrich Japan, Tokyo, Japan) for $20 \mathrm{~min}$ to dissolve SBA15, washed with ethanol (Sigma-Aldrich Japan, Tokyo, Japan), and dried in air at $60^{\circ} \mathrm{C}$ for $12 \mathrm{~h}$.

\subsection{Characterization}

The prepared sample was examined using a powder X-ray diffractometer (X'Pert-PRO, Malvern PANalytical, Tokyo, Japan) with $\mathrm{Cu} K \alpha$ radiation $(\lambda=0.154060 \mathrm{~nm})$. A non-reflection Si plate was used as a sample holder to reduce the background. Hitachi SU8200 scanning electron microscope equipped with an X-ray energy-dispersive spectrometer (Hitachi, Tokyo, Japan) was used for microscopic characterization and elemental compositions at an accelerating voltage of $5 \mathrm{kV}$ and $15 \mathrm{kV}$, respectively. High-resolution transmission electron microscopic (HRTEM) images were obtained with a JEOL 2100-F2 microscope (JEOL, Tokyo, Japan).

\section{Results and Discussions}

Figure 1a shows the $p$ XRD profiles of the prepared nanoporous Au-Fe alloy $(20<2 \theta<90$ degrees). The major reflection peaks were positioned at $2 \theta=38.80,45.10,65.60,78.90$, and 83.06 degrees, which corresponded to the (111)-, (200)-, (220)-, (311)-, and (222) of the face-centered cubic structure ( $a=0.3980 \pm 0.0002 \mathrm{~nm}$ ), respectively. The chemical composition of the Au-Fe alloy was estimated as $\mathrm{Au}_{0.75} \mathrm{Fe}_{0.25}$ because the calculated lattice parameter was consistent with the reported value of an atomically ordered $\mathrm{Au}_{3} \mathrm{Fe}$ alloy [10]. The peak positions were shifted toward the higher reflection angles with respect to the corresponding peaks from pure $\mathrm{Au}$ at 38.10, 44.30, 64.50, 77.50, and 81.60 degrees $(a=0.4070 \pm 0.0002 \mathrm{~nm})$, showing that the crystal lattice of the nanoporous Au-Fe was $2.2 \%$ smaller than that of pure Au due to the incorporation of Fe atoms in the Au matrix [10]. The low-angle $p$ XRD profile of the nanoporous Au-Fe $(0.70<2 \theta<2.0$ degrees $)$ is presented in Figure $1 \mathrm{~b}$. There are two peaks observed at $2 \theta=0.80$ and 1.30 degrees, corresponding to the (100)- and (110) reflections of a hexagonally ordered nanopores replicated from SBA15, of which lattice parameter along the hexagonal edge was calculated as $13.20 \pm 0.02 \mathrm{~nm}[13,14]$.

(a)

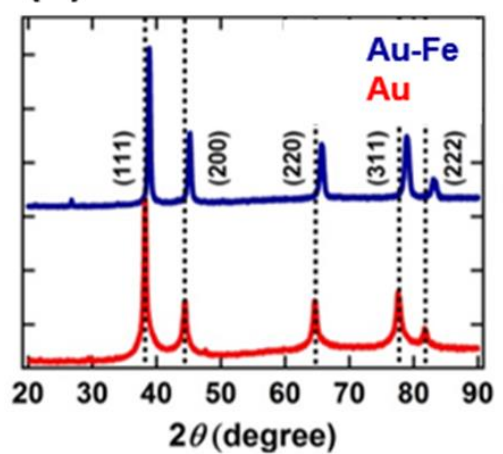

\section{(b)}

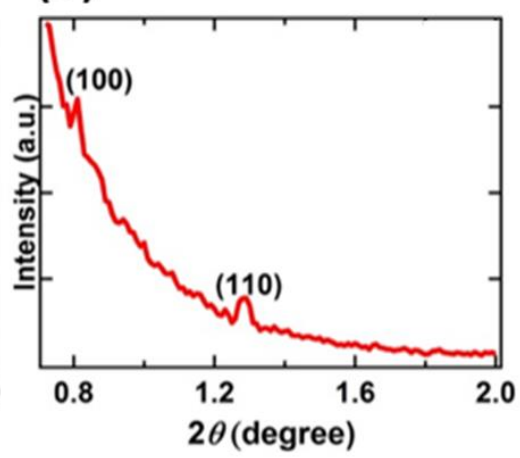

Figure 1. $p \mathrm{XRD}$ profiles for Au nanoparticles and the nanoporous Au-Fe alloy in high reflection (a) and low reflection angles $(\mathbf{b})$.

Figure 2 presents the nitrogen $\left(\mathrm{N}_{2}\right)$ adsorption-desorption isotherms of the nanoporous $\mathrm{Au}-\mathrm{Fe}$. An aliquot of $100 \mathrm{mg}$ of sample was degassed at $150{ }^{\circ} \mathrm{C}$ for $3 \mathrm{~h}$, then the $\mathrm{N}_{2}$-adsorption/desorption isotherm was carried out using Micrometrics ASAP 2020 (National Institute for Materials Science (NIMS), Tsukuba, Japan). The specific surface area of the nanoporous Au-Fe was calculated as $81.2 \mathrm{~m}^{2} \cdot \mathrm{g}^{-1}$ from the $\mathrm{N}_{2}$ adsorption-desorption isotherms. The pore-size distribution curve (inset in 
Figure 2) had a maximum at $18 \mathrm{~nm}$, which was consistent with the lattice constant (13.2 $\mathrm{nm})$ of the ordered nanopores that was determined by low-angle $p$ XRD (Figure 1b). The mean value of the pore diameter was calculated from the $\mathrm{N}_{2}$ adsorption data as $24 \pm 2 \mathrm{~nm}$.

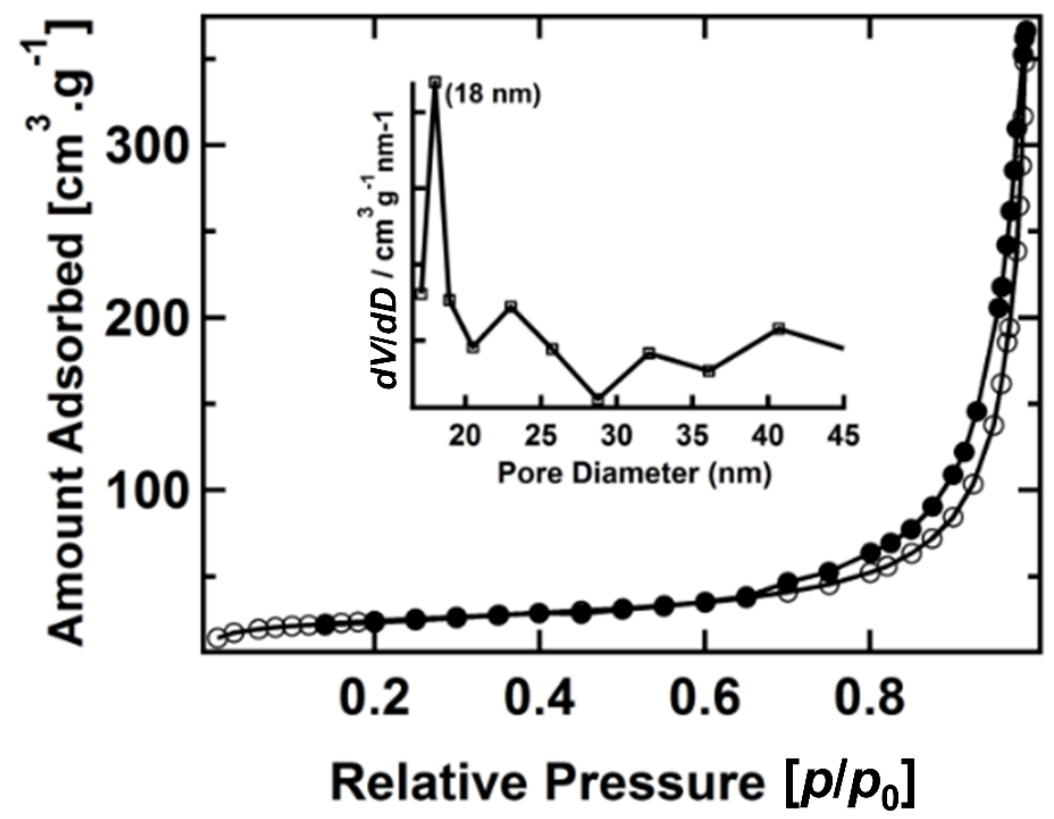

Figure 2. $\mathrm{N}_{2}$ adsorption-desorption isotherms for the Au-Fe nanomaterials (open symbols: adsorption, closed symbols: desorption). Inset indicates the pore-size distribution.

The successful replication of the nanoporous structure of the SBA15 template to the nanoporous Au-Fe was confirmed by microscopic characterizations including SEM and TEM (Figure S1). Figure 3a shows that the prepared nanoporous Au-Fe had a rod-like shape with an average thickness of $1 \pm 0.1 \mu \mathrm{m}$, having a number of pores over the surface (Figure 3b). High-resolution TEM observation demonstrated that the porous structure was developed not only over the surface but also in the bulk, of which pore diameter was 10-20 nm (Figure 3c). Compositional mapping showed that Au and Fe were uniformly distributed in the nanoporous $\mathrm{Au}-\mathrm{Fe}$ (the sample was finely pulverized by sonication in ethanol to improve electron-beam transmission for quantitative analysis). Based on the results of $p$ XRD, SEM (JEOL, Tokyo, Japan) and TEM (JEOL, Tokyo, Japan) characterizations, we conclude that the nanoporous $\mathrm{Au}$-Fe comprised hexagonally ordered nanopore arrays of $\mathrm{Au}_{0.75} \mathrm{Fe}_{0.25}$ alloy (see Figures S2-S5, Table 1 for HAXPES and EDS data). We further estimated the composition of the Au-Fe nanomaterials from the HAXPES peak intensities of metallic Au $4 f$ - and Fe $2 p$ emissions (Figures S2-S4). The calculated Au/Fe ratio was $2.42 \pm 0.24$ (see supporting information), which is fairly consistent with the value estimated from $p$ XRD: $\mathrm{Au} / \mathrm{Fe}=0.75 / 0.25=3$. 

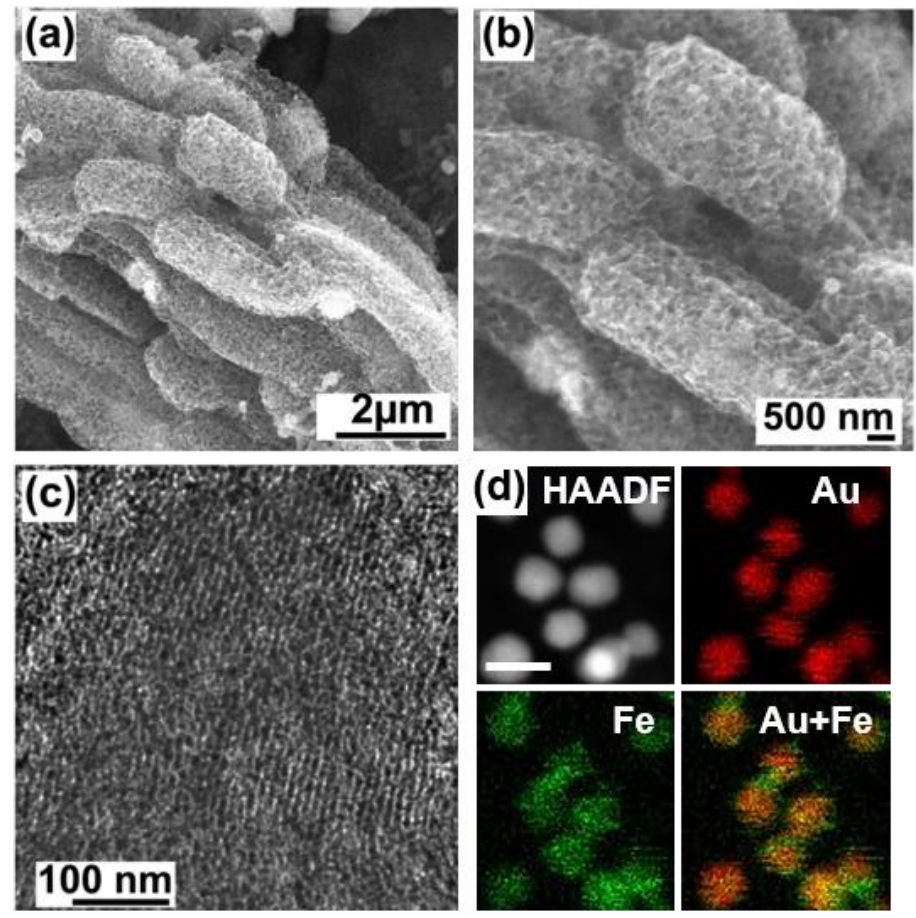

Figure 3. (a) Scanning electron microscope (SEM); and (b) transmission electron microscope (TEM) images of the nanoporous Au-Fe (c). An annular-dark field (HAADF) image and compositional mapping images of the nanoporous $\mathrm{Au}-\mathrm{Fe}$ corresponding to $\mathrm{Au}, \mathrm{Fe}$, and a merged image (d). The scale bar in (d) corresponds to $10 \mathrm{~nm}$.

To verify how the hard template contributed to the homogeneity and porous nature of the nanoporous $\mathrm{Au}-\mathrm{Fe}$, free-standing $\mathrm{Au}-\mathrm{Fe}$ nanoparticles were synthesized without SBA15 by co-reduction of the same precursors in the same condition as for the nanoporous $\mathrm{Au}-\mathrm{Fe} . \mathrm{AuCl}_{3}$ and Fe(II)acac were dissolved in diglyme containing no SBA15 and reduced with superhydride to yield black, fine powder of Au-Fe nanoparticles. The Au-Fe nanoparticles were post-annealed in vacuum at $700{ }^{\circ} \mathrm{C}$ as was the nanoporous $\mathrm{Au}-\mathrm{Fe}$. The obtained nanoparticles were widely distributed in size from $20 \mathrm{~nm}$ to $100 \mathrm{~nm}$ (Figure 4a). Moreover, the chemical composition of the prepared nanoparticles was not uniform: each of the individual alloy nanoparticles had different Au and Fe contents (Figure 4b).
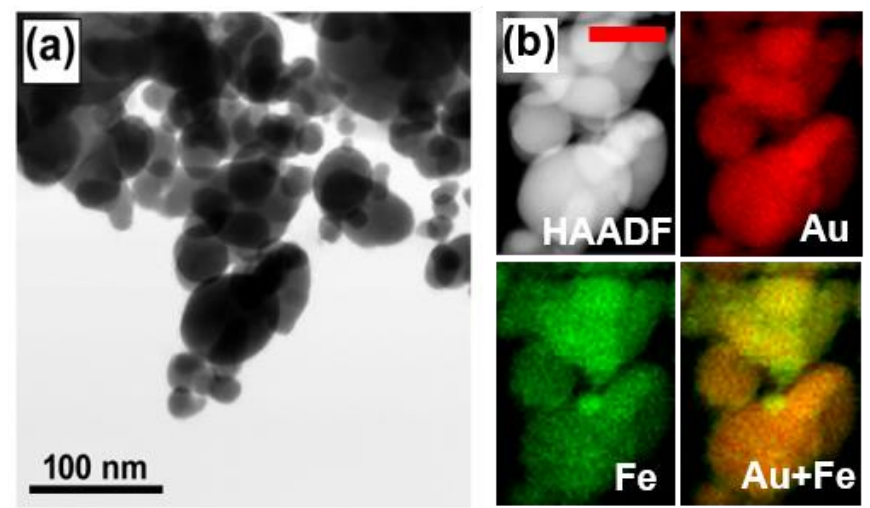

Figure 4. (a) A transmission electron microscope (STEM) image annular-dark field (HAADF) image and (b) compositional mapping images of the Au-Fe nanoparticles synthesized without templates. The red scale bar in (b) corresponds to $50 \mathrm{~nm}$. 
The low homogeneity in size and composition of the Au-Fe nanoparticles most likely resulted from the uncontrolled reduction kinetics of the precursors. The $\mathrm{Au}(\mathrm{III})$ - and $\mathrm{Fe}(\mathrm{II})$ precursors were reduced by Super-Hydride to randomly form alloy embryos of different sizes and compositions in solvent. The post-annealing processes improved neither the compositional homogeneity nor the size distribution of the individual nanoparticles. Unlike the free-standing nanoparticles, the nanoporous Au-Fe alloy exhibited a highly uniform pore distribution and homogenous composition because the nanoporous SBA15 acted as a scaffold that absorbed the different precursors. The Au(III)- and Fe(II) precursor molecules were, as depicted in Scheme 1, atomically dispersed over the nanopore surface of SBA15. The adsorbed precursor molecules were then chemically reduced and vacuum-annealed below the phase-separation temperature to form skeletal, nanoporous Au-Fe alloy materials that were uniform in pore size and chemical composition.

Different nanoporous metals are efficiently used for catalysis, energy conversions, and sensors because of the large specific surface areas $\left(>100 \mathrm{~m}^{2} \cdot \mathrm{g}^{-1}\right)$ and high pore volumes $\left(>1 \mathrm{~cm}^{3} \cdot \mathrm{g}^{-1}\right)[15,16]$. Most of the nanoporous metals materialized consisted of almost pure precious metals such as gold ( $\mathrm{Au})$. Nanoporous Au can be materialized through different pathways including dealloying, sputtering, and implanting. However, the title material of our paper, Au-Fe nanomaterials, are hardly materialized through traditional processes because of the large difference in reduction potential of the constituent $\mathrm{Au}$ and $\mathrm{Fe}$. Fe is readily oxidized to form iron oxides, whereas Au always retains the inherent metallic nature.

In conclusion, metastable Au-Fe alloy was successfully synthesized in the form of nanoporous materials (nanoporous $\mathrm{Au}-\mathrm{Fe}$ ) via a wet chemistry route using SBA15 as a hard template. The nanoporous Au-Fe comprised hexagonally ordered arrays of nanopores that were uniform in pore diameter (10-20 nm in diameter) and chemical composition $\left(\mathrm{Au}_{0.75} \mathrm{Fe}_{0.25}\right)$. The effectiveness of the hard template was demonstrated by a control synthesis of free-standing Au-Fe nanoparticles, which were widely dispersed in size and composition. The successful hard-template synthesis of the nanoporous Au-Fe can be applied to a broad range of alloy systems and will open up unexplored research fields on functional alloy nanomaterials such as high-performance catalysts and adsorbents, which will have high specific surface areas, controllable surface-atom arrangements, and uniformly distributed nanoporous structures.

Supplementary Materials: The following are available online at www.mdpi.com/2075-4701/8/1/17/s1. Figure S1: Scanning Electron Microscope (SEM) images of the nanoporous AuFe and Silica template, Figure S2: Hard X-ray Photoemission Spectroscopy (HAXPES) in a wide range of binding energy, Figure S3: HAXPES in the $\mathrm{Au} 4 \mathrm{f}$ region, Figure S4: HAXPES in the Fe 2p region, Figures S5: Energy-dispersion Spectroscopy (EDS) analyses on the nanoporous Au-Fe, Table S1: Chemical composition of the nanoporous Au-Fe determined by EDS.

Acknowledgments: This work was preliminarily supported by the JST CREST. This work was financially supported by JST CREST (Japan Science and Technology Agency: JST) and NIMS microstructural characterization platform as a program of the "Nanotechnology Platform" of the Ministry of Education, Culture, Sports, Science and Technology (MEXT), Japan. The HAXPES measurements were performed under the approval of the NIMS Synchrotron X-ray Station (Proposal No. 2017A4602 and 2016B4600). The authors are grateful to HiSOR, Hiroshima University and JAEA/SPring-8 for the development of HAXPES at BL15XU of SPring-8.

Author Contributions: Paskalis Sahaya Murphin Kumar designed the work, synthesized the material, and conducted all the characterizations. Takeshi Fujita conducted TEM characterizations. Hideki Abe, Ramasamy Jayavel, and Thiripuranthagan Sivakumar worked together to complete the manuscript.

Conflicts of Interest: The authors declare no conflict of interest.

\section{References}

1. Rick, J.; Tsai, M.C.; Hwang, B.J. Biosensors incorporating bimetallic nanoparticles. Nanomaterials 2016, 6, 5. [CrossRef] [PubMed]

2. Pannu, C.; Bala, M.; Khan, S.A.; Srivastava, S.K.; Kabiraj, D.; Avasthi, D.K. Synthesis and characterization of Au-Fe alloy nanoparticles embedded in a silica matrix by atom beam sputtering. RSC Adv. 2015, 5, 92080-92088. [CrossRef] 
3. Yan, Y.C.; Du, J.S.S.; Gilroy, K.D.; Yang, D.R.; Xia, Y.N.; Zhang, H. Intermetallic nanocrystals: Syntheses and catalytic applications. Adv. Mater. 2017, 29. [CrossRef] [PubMed]

4. Liu, S.; Chen, G.; Prasad, P.N.; Swihart, M.T. Synthesis of monodisperse Au, Ag, and Au-Ag alloy nanoparticles with tunable size and surface plasmon resonance frequency. Chem. Mater. 2011, 23, 4098-4101. [CrossRef]

5. Lehoux, A.; Ramos, L.; Beaunier, P.; Uribe, D.B.; Dieudonne, P.; Audonnet, F.; Etcheberry, A.; Jose-Yacaman, M.; Remita, H. Tuning the porosity of bimetallic nanostructures by a soft templating approach. Adv. Funct. Mater. 2012, 22, 4900-4908. [CrossRef]

6. Oezaslan, M.; Hasche, F.; Strasser, P. Pt-based core-shell catalyst architectures for oxygen fuel cell electrodes. J. Phys. Chem. Lett. 2013, 4, 3273-3291. [CrossRef]

7. Chen, H.; Wang, D.L.; Yu, Y.C.; Newton, K.A.; Muller, D.A.; Abruna, H.; DiSalvo, F.J. A surfactant-free strategy for synthesizing, and processing intermetallic platinum-based nanoparticle catalysts. J. Am. Chem. Soc. 2012, 134, 18453-18459. [CrossRef] [PubMed]

8. Chen, H.; Yu, Y.C.; Xin, H.L.L.; Newton, K.A.; Holtz, M.E.; Wang, D.L.; Muller, D.A.; Abruna, H.D.; DiSalvo, F.J. Coalescence in the thermal annealing of nanoparticles: An in situ stem study of the growth mechanisms of ordered Pt-Fe nanoparticles in a KCl matrix. Chem. Mater. 2013, 25, 1436-1442. [CrossRef]

9. Malgras, V.; Ataee-Esfahani, H.; Wang, H.J.; Jiang, B.; Li, C.L.; Wu, K.C.W.; Kim, J.H.; Yamauchi, Y. Nanoarchitectures for mesoporous metals. Adv. Mater. 2016, 28, 993-1010. [CrossRef] [PubMed]

10. Bondi, J.F.; Misra, R.; Ke, X.L.; Sines, I.T.; Schiffer, P.; Schaak, R.E. Optimized synthesis and magnetic properties of intermetallic $\mathrm{Au}_{3} \mathrm{Fe}_{1-x}, \mathrm{Au}_{3} \mathrm{Co}_{1-x}$, and $\mathrm{Au}_{3} \mathrm{Ni}_{1-x}$ nanoparticles. Chem. Mater. 2010, 22, 3988-3994. [CrossRef]

11. Naitabdi, A.; Cuenya, B.R. Formation, thermal stability, and surface composition of size-selected aufe nanoparticles. Appl. Phys. Lett. 2007, 91, 113110. [CrossRef]

12. Zhuravlev, I.A.; Barabash, S.V.; An, J.M.; Belashchenko, K.D. Phase stability, ordering tendencies, and magnetism in single-phase fcc Au-Fe nanoalloys. Phys. Rev. B 2017, 96, 134109. [CrossRef]

13. Kruk, M.; Jaroniec, M.; Ko, C.H.; Ryoo, R. Characterization of the porous structure of SBA-15. Chem. Mater. 2000, 12, 1961-1968. [CrossRef]

14. Margolese, D.; Melero, J.A.; Christiansen, S.C.; Chmelka, B.F.; Stucky, G.D. Direct syntheses of ordered SBA-15 mesoporous silica containing sulfonic acid groups. Chem. Mater. 2000, 12, 2448-2459. [CrossRef]

15. Seker, E.; Reed, M.L.; Begley, M.R. Nanoporous Gold: Fabrication, Characterization, and Applications. Materials 2009, 2, 2188-2215. [CrossRef]

16. Ding, Y.; Kim, Y.-J.; Erlebacher, J. Nanoporous Gold Leaf: “Ancient Technology"/Advanced Material. Adv. Mater. 2004, 16, 1897-1900. [CrossRef]

(C) 2017 by the authors. Licensee MDPI, Basel, Switzerland. This article is an open access article distributed under the terms and conditions of the Creative Commons Attribution (CC BY) license (http:/ / creativecommons.org/licenses/by/4.0/). 